\title{
Téoros
}

Revue de recherche en tourisme

\section{Parcs, tourisme et développement durable}

\section{André Barabé}

Volume 14, numéro 1, printemps 1995

Les parcs : des réseaux en mutation

URI : https://id.erudit.org/iderudit/1077048ar

DOI : https://doi.org/10.7202/1077048ar

Aller au sommaire du numéro

Éditeur(s)

Université du Québec à Montréal

ISSN

0712-8657 (imprimé)

1923-2705 (numérique)

Découvrir la revue

Citer cet article

Barabé, A. (1995). Parcs, tourisme et développement durable. Téoros, 14(1),

8-12. https://doi.org/10.7202/1077048ar d'utilisation que vous pouvez consulter en ligne.

https://apropos.erudit.org/fr/usagers/politique-dutilisation/ 


\title{
Parcs, tourisme et développement durable
}

\author{
André Barabé
}

Les pares nationaux et provinciaux comptent parmi le patrimoinecollectifnaturel placésous la tutelle de l'Etat. La gestionde ce bien collectif́ne va pas sans problème et exige de concilier deux objectifs dualistes. D'une part, les parcs doivent être entretenus et protégés de façon à être transmis intactsauxgénérations futures. Cetteobligation morale et légale traduit bien la conviction qu'il s'agit d'un héritage que nous devons léguer à nos enfants. D'autre part, les parcs exercent une forte attraction sur les populations urbaines contemporaines. Les parcs sont perçus comme un gisement naturel exceptionnel qui suscite de fortes demandes pour la récréation et le tourisme. Voila le paradoxe et le défi associés à la conservation et a la mise en valeur des parcs dans une perspective de développement durable.

L'objet de l'article est de comprendre les principales tendances et enjeux qui affectent l'évol ution des parcs et du tourisme de nature qui y est associé. Cet exercice de synthèse devrait également permettre de mieux situer la mission des parcs, en tant qu'aires protégées, au sein de la Stratégie mondiale de la conservation pour le développement durable.

\section{Accessibilité : du tourisme de masse à l'écotourisme \\ Du tourisme de masse au loisir de proximité}

Dédiés à la population pour son bénéfice, son instruction et sa jouissance, les parcs sontrapidement devenusdes sites d'attraction touristique. Les villages hôteliers, développés à l'intérieur des limites des parcs nationaux de Banff et de Jasper, témoignent du caractere favorable des premieres politiques de gestion à l'egard du tourisme. Après un peuplus decentans, on constate que le tourisme de masse menace l'intégrité écologique des aparcs naturels vedettess. Une enquête réconte menée aupres de 70 sites naturels, classés sur la listedu Patrimoinemondial del'UNESCO,

Monsieur André Barabé est professeur au département des sciences du koisir à I'Universite du Quebec à Trois-Aivières. révèle que ces sites reçoivent plus de quarante millions de visiteurs par an (Sources: UNESCO, 1994). D'autre part, le pare *Great Smoky Mountains" reçoit 9 millions de visiteurs par an, tandis que le parc Yellowstone accueille annuellement 3 millions de visiteurs. Ces sites vedettes sont victimes de leur notoriété internatiomale. Tout en reconnaissant quele tourisme de masse représente un problème majeur, lés responsables interrogés ne se plaignent pasde la fréquentation qui représente pour eux un apport financier essentiel.

La situation qui prévaut dans les réseaux canadiens et québecois s'avère fort différente. Les chiffres de fréquentation précédemment mentionnés ne sont en aucune façon comparables avec la réalité des pares sur le territoire québécois. Le déferlement des hordes de touristes étrangers qui détruisent les ressources naturelles de nos parcs tient plus de la fiction que de la réalité. En 1993, un peuplus de 14000000 devisiteursont fréquentél'entierdu réseau des pares nationaux canadiens. De ce nombre, 442091 visiteurs ont été enregistrésdans les trois parcsnationaux terrestres situés au Québec: parc de la Mauricie (247329 visites), Forillon (169412 visites), Archipel-de-Mingan (25350 visites) (Parcs Canada, Rapport Statistique 1993). Pour leur parr, les 17 parcs du réseau québécois ont reçu quelque 2655680 visites entre le mois d'avril et le mois d'octobre 1994. La moyenne de fréquentation par pare se chiffreà 156217 visites. Cettemoyennene rend toutefois pas compte des situations extrêmes: Mont-Tremblant (547 533), Mont-Mégantic (3047) (Fréquentation mensuelle dans le résenu des parcs, DPAP, 1904).

Ces chiftes de fréquentation montrent que les parcs situés sur leterritoirequébécois ne s'inscrivent pas dans la logique du tourisme de masse affectane les pares nationaux des Etats-Uniset del'Europe (Richez, 1992). La réalité québécoise de la fréquentation des parcs tient davantage à la promotion du récréo-tourisme. Les premiers enjeux socinux de la politique du loisir a porté sur l'acoessibilité aux lieux de plein air et aux parcs (Livre Blanc sur le loisir au Québec, 1979). Cette perspective nous permet de bien saisir la raison de l'existence dedeuxcatégories de parcsdans le réseau québécois: les pares de récréation et les parcs de conservation.

L'accessibilité aux activités de plein air étant un critère majeur pour la localisation des parcsde récréation, il n'est pasétonnant que ces parcs de cette catégorie soient situésdans la périphérie de la grande agglomération montréalaise (sauf le pare Frontenac). Ces parcs recueillent $61 \%$ des visites du réseau. Si on y ajoute la fréquentation du parc du Mont Saint-Bruno, classifié comme parc de conservation mais localisé dans le voisinage immédiat de Montréal, on constate que 7 pares sur 17 cumulent $74 \%$ des visites. De plus, ces visites se concentrent essentiellement en juillet et août, puis en septembre et juin. Ainsi, les parcs de récréation se caractérisent par un loisir de proximité. La fréquentation qui en émane se distingue pas sa forte concentration dans l'espace et dans le temps. D'autre part, si l'on remarque une congestion des espaces les plus accessibles, on observe également que les autres parcs du réseau souffrent d'une sous-utilisation. Il s'agit généralement des parcs de conservation, qui dans la logique de «représentativité des régions naturelless, sont disśminés sur l'ensemble du territoire québécois et éloignés des concentrations urbaines.

Du ré̉créc-tourisme à l'écotourisme Les pares de conservation sont créés pour assurer la protection permanente de territoires représentatifs des régions naturelles du Québec ou de sites naturels à caractère exceptionnel. Ils sont accessibles au public pour des fins d'éducation au milieu naturel (MLCP, La politique, 1982). Si l'enjeu premier des pares de récréation était d'accroitre l'accessibilité aux lieux de plein air, l'enjeu des parcs de conservation est d'offrir uneexperienced'observation et d'érude au sein d'une nature sauvage de grande qualité. La priorité ne porte pas sur l'activité récréative et l'importance des infras- 
tructures de services, mais bien sur la nature comme valeur intrinsèque.

Les chiffres de fréquentation, précédemment cités, indiquent que les parcs de conservation sont peu utilisés. De même, une étuderécenteportantsur le positionnement des parcs et des réserves fauniques du Québec montre que ces parcs sont méconnus du public (Laliberté et Lanctôt, Coopers et Lybrand, 1992). Pourtant, les parcs de conservation s'inscrivent dans un créneau national et international en pleine expansion: celui de l'ćcotourisme. Le tourisme écologique représente environ $5 \%$ du marché touristique total. Toutefois, le créneau porteur de l'écotourisme croît de $20 \%$ à $25 \%$ par année (Blangy, 1993), alor's que l'on prévoyait une croissance annuelle de $10 \%$ à $15 \%$ (Lindherg, 1991) au début de la décennie.

L'écotourisme représente une opportunité de positionnement intéressante pour les parcs de conservation. Il est considéré comme le tourisme idéal pour les parcs nationaux et les zones protégées (CCCE, 1991). Cetteposition du Conseil consultat if canadien de l'environnement s'appuie sur les caractéristiques actuelles du tourisme écologique. En premier lieu, l'écotourisme exige des environnements naturels dehaute qualité. On recherche des aires protégées peu ou pas perturbées, présentant une grande diversité biologique et des aménagements légers. En second lieu, l'écotourisme aocorde une grande attention au caractère éducatif des expériences vécues. On souhaite disposer d'une information bien conçue et d'une bonne qualité d'encadrement de la part de guides maturalistes pouvant faciliter l'exploration et la connaissancedu milieu. En troisième lieu, cette formealternative de tourisme est réputée être respectueuse et soucieuse de minimiser les impacts sur $l^{*}$ environnement et les communautés d'accueil. Enfin, l'écotourisme peut créer des retombées économiques intéressantes pour les collectivités locales vivant en périphérie des parcs. Le développement del'écotourisme possède cependant sa contrepartie. Il est impératif pour les gestionnaires d'éviter de répéter la légende du cheval de Troie, en introduisant volontairement, au sein des milieux naturels les plus sensibles et lesplus vulnérables les acteurs qui ouvrent la porte à la destruction da milieu même que l'on voulait protéger. En conséquence, il faut anticiper certaines mesures de contrôle et une limitation du nombredepersonnespar groupe pratiquant des activités éotouristiques dans les zones fragiles.

Quelques activités d'interprétation, présentement disponibles dans certains parcs de conservation du Québec, offrent un bon potentiel pour le développement d'expériences écotouristiques. Nous pensons particulièrement aux randonnées guidées pour l'observation du caribou dans le parc des Grands-Jardins et dans le parc de la Gaspésie; l'appel aux loups dans le parc de la Jacques-Cartier; l'observation des baleines dans le parc marin du Saguenay. Ces expériences peuvent servir de point d'appui pour la conception et la promotion d'un produit d'appel écotouristiqueservant de positionnement aux pares de conservation. La mise en forfait de tels produits pourrait d'abord s'adresser au segment de marchédu tourisme de nature interrégional etnational. Egalement, quelques produits écotouristiques vedettes seraient susceptibles de retenir l'attention du segment de marchéinternationaldu tourismede nature, particulièrement sensible aux grands espaces sauvages et aux espèces fauniques fragiles ou menacées. Toutefois, une telle entreprise ne peut être menée à bien sans un partenariat ef un partage des responsabilités entre l'Etat, l'ind lustric touristique et les collectivitis loc:iles.

\section{Conservation : de l'isolation à l'intégration}

\section{Menaces internes et externes}

La façon de concevoir et de gérer la pérennité des ressources s'est considérablement modifiće au cours des cent dernières années. La stratégie de préservation du XIX' siécleconsistait à créer degrands espaces quel ion voulait protéger contre l'exploitation commerciale des ressourcesnaturelles, L'intentionsous-jacente consistait ì consetver intégralement les écosystemes initiaux, en les plagant en quelque sorte sous globe et isolés des influences extéritures (contrôle du braconnage, des prédateurs, des feux de forêts, des épidémies d'insectes). La stratégie de conservation du $X^{t}$ siècle consiste davantage à vouloit préserver l'évolution naturelle des parcs plutôt que son aspect initial. En ćtant constient que le milieu biophysique est une rúalicé en mutation, il ne s'agit plusici d'en fixer l'évolution. D'une conception statique de la préservation, on passe ì une conception dynamique. Cette évolution de la problématiquedu maintien de la pérennité des ressourcess'accompagneégalement de nouveaux défis.

Ces défis portent sur le contrôle des menaces internes et des menaces externes qui affectent la diversité des espèces et des ressources des parcs. Au cours des dernières décennies, la lutte aux menaces internes a surtout retenu l'attention des gestionnaires. Ces pressions intérieures viennent principalement des utilisateurs qui fréquentent ces territoires. Les impacts de l'utilisation récréative sur les ressources naturelles sont nombreux: impacts sur les sols et sur la végétation, dérangement de la faune, vandalisme sur les infrastructures. Dans cette perspective, les gestionnaires des pares ont développé des stratégies et desmoyens d'action efficaces pour oontrôler la circulation des visiteurs et minimiser les impacts sur les ressources naturelles. A titre d'exemple, mentionnons l'élaboration de plans directeurs d'aménagement, de plans de conservation des ressources naturelles, de programmes d'évaluation environnementale et de processus de suivi des impacts, méthodologies d'évaluation des capacités de charge, etc.

Les nouveaux défis prennent davantage la forme de menaces extérieures exercées sur les écosystemes et les processus naturels des parcs. Il s'agit de pressions reliées à l'utilisation des terres avoisinantes. Ainsi, les coupes forestières pratiquées dans les zones limitrophes des parcs sont susceptibles d'affecter la végétation et la faune à l'intérieurdesparcs. Lesmenaces d'origines internationales, telles les pluies acides, affectent particulièrement certains pares du Québec (par exemple, le parc national de la Mauricie). Quoiqu'il en soit, la prise en compte des menaces internes et externes s'avìre nécessaire afin d'assurer l'intégrité écologique des parcs.

Pares et concept d'intégrité écologique Par le passé, la protection des ressources naturelles s'est effectuée sur une base micro-spatiale. Les interventions de nature ponctuelle ont été orientées d'une part, vers la réhabilitation des sites affectés par desdégradationsantérieuresetd'autrepart, vers la réduction des impacts associés à l'implantation d'infrastructures et à la pratiq̨ue d'activirśs récréatives. Les tendances actuelles en matière de gestion des ressources naturelles s'inspirent davantage d'une approche macro-spaciale. Fondés sur une approche ecosystémique, les conceptions et les moyens mis en oeuvre pour assurer la 
pérennité des ressources semblent se globaliser et se situer à l'échelle des écosystèmes. Ce changement d'approche se reflete bien dans l'adoption du concept d'intégrité écologique qui occupe la première place des principes-directeurs dans la gestion des parcs nationaux canadiens (Pares Canada, 1994).

La création d'espaces naturels protégés a longtemps servi de caution morale pour justifier une exploitation intensive des ressources naturelles ailleurs sur le territoire et souvent même, en périphérie des parcs et réserves naturelles. En conséquence, il ne faut pas s'étonner que plusieurs parcs soient devenus des *ilots de protection $*$ au milieu de vastes territoires humanisés. La notion d'intégrité écologique oblige à dépasser la vision des pares comme etant des entités écologiquement autosuffisantes et indépendantes desterritoires adjacents. Au contraire, les parcs font partied'écosystèmes et de paysages culturels qui les englobent. De plus en plus, l'intégrité même des ressources naturelles des parcs dépendent des influences extérieures qui les menacent et sur lesquelles les parcsne possèdent aucune juridiction. Ces influences externespeuvent relever de la gestion des terres(exploitation forestière, exploitation faunique, fragmentation d'habitats, modification des structures de populations, dérangement de la faune) ou de perturbations naturelles (régime hydrique, feu naturel, épidémie d'insectes) quiont des répercussions sur les espèces, lescommunautéset les éonsystèmes des parcs(Foisy, 1994). Ledéveloppement d'ententes de coopération avec les gestionnaires des terres avoisinantes apparaît prioritaire pour l'avenir.

Quelles seront les conséquences de la valorisation du concept d'intégrité écologique sur l'accessibilité et l'accueil touristique des parcs? D'une part, cette approche ouvre de belles perspectives de partenariatavec les gestionnairesdes terres adjacentes et avec les intervenants touristiques. D'autre part, il n'est pas abusif d'anticiper une remise en question de certaines activités (droits de pêche sur certains lacs, etc.) et de certains services (hếbergement, etc) à l'intếrieur des parcs, ainsi que l'intensification de certaines mesures de régulation. L'objectif de conserver l'intégrité écologique des parcs, au moment où la demande pour le tourisme de nature est en pleine expansion, repré- senteun défi de taille pour les gestionnaires de pare.

\section{Parcs et développement durable}

Leconcept de développement durablepeut être circonscrit à partir de quelques caractéristiques centrales. En premier lieu, on rappelle que la croissance et le développementont pour but primordial la satisfaction des besoins essentiels des communautés humaines. En second lieu, on précise que la compatibilité entre développement et environnement est conditionnelle au maintien de la pérennité des ressources vivantes et au respect de la capacité de charge des écosystèmes. En troisièmelieu, on insistesur l'importance dusouci d'équité dans l'utilisation des ressources naturelles entre les générations actuelles et les générations futures. En quatrième lieu, on constate que la philosophie du développement durable fait appel ì une nouvelle orientation de notre penséeet impliquedes changements de valeurs, d'attitudes et de comportements. Sur la base de ces principes-clés, nous tenterons maintenant de résumer la contribution et les défis attribués aux parcs en lien avec la stratégie du développement durable.

\section{Pérennité des ressources}

\section{naturelles vivantes}

En premier lieu, force est de reconnaittre la contribution exceptionnelle des pares au maintien de la pérennité des ressourees naturelles. Les parcs ont joué un rôle précurseur en sélectionnant et protégeant des échantillons représentatifs d'écosystèmes régionaux terrestres et marins. Dans les limites de leur mandat, les pares contribuent directement à la sauvegarde de la diversité biologique des écosystèmes, des espèces et de leurs habitats sur leur territoire de référence. La protection d'espèces menacées ou vulnérables est un autre motif qui a servi de justification à la création de parcs (hison, caribou, béluga, etc.). La préservation du patrimoine génétique est un rôle que les parcs de conservation pourraient davantage assumer à long terme. Cette orientation s'inscrit très bien dans les tendances internationales actuelles et constitue une réponse concrète aux obligations du Canada et du Québec, signataires de la Convention internatiomale sur la diversité biologique proposće par les Nations Unis en 1902.

Les actuis actuels sont impressionnants mais encore insuffisants. Les superficies protégées, les fréquentations actuelles et les infrastructures développées représentent d'excellents points. Les pratiques de gestion visant à limiter les impacts des visiteurs sur les ressources naturelles sont bien documentées et font l'objet d'interventions efficaces sur le terrain. Par contre, la confection de plans de conservation, visantl'étude systématiquedes écosystèmes et de leur évolution naturelle dans un contexte régional, sont encore peu développés. Le défi de l'intégrité écologique, incluant la maîtrise des menaces internes et externes, risque d'occuper la première place dans l'avenir en ce qui a trait à la gestion des ressources naturelles.

Par ailleurs, il apparait primordial de compléter les immobilisations en latence et d'achever les réseaux de pares nationaux et provinciauxsur la base de la représentativité des régions naturelles biogéographiques. Il faut tendre vers l'objectif de $12 \%$ de terres protégées, proposé par la Commission mondiale sur l'environnement et le développement (1987) et endossé par le Canada (1990). Bien que pionniers en matière de conservation, les parcs doivent maintenant composer avec d'autres types d'aires protégées et s'intégrer dans la Stratégie mondiale de conservation et de développement durable (UICN, PNUE, WWF, 1980; CMED, 1987; Sommet de la Terre, 1992). Dans le contexte des enjeux planétaires, la mosiąque internationale des pares est susceptible de jouer un rôle majeur dans l'analysemacro-écologiquereliée au maintien des processus écologiques essentiels et des cycles biosphériques.

\section{Equité sociale et accessibilité}

Le développement durable implique un souci d'équité sociale entre les générations (CMED, 1989). Ce principe fondamental met l'accent surl'accessibilité et un partage équitable des coûts et des bénéfices de l'utilisation des ressources et de la conservation de l'environnement entre les générations actuelles et les générations futures.

Leprincipe de l'équitésociale n'est pas une problématique nouvelle pour les gestionnaires de territoires protégés. $\mathrm{La}$ politique des parcs nationaux canadiens et des parcs provinciaux québécois précise que les parcs doivent être entretenus et utilisés pour le bénéfice des générations actuelles et futures. Répondant à cette mission, lesorientations degestion del'Etat ont soutenu l'accessibilité physique et financièreaux parcs. La création de parcsen périphérie des grandes agglomérations ur- 
baines et la mise en place d'infrastructures d'accueil et de services témoignent de ces efforts. Egalement, une faible tarification pour les activités et les services reflète cet engagement à offrir «une chance égale à tous\%. En somme, le rapport qualité-prix pour le «produit parc» est généralement perçu comme supérieur aux autres sites de plein air (Laliberté et Lanctôt, Coopers et Lybrand, 1992).

Cependant, la situation passée n'est pas garante del'avenir. Au contraire, les acquis obtenus au prix de nombreuses années d'efforts sontaujourd'hui menacés et remis en question. Les compressions budgétaires répétées forcent les ministères responsables des parcsà définir de nouvelles priorités et stratégies d'action. Le nouveau cadre de référence implique une politique d'autofinancement et de délégation de gestion. En corollaire, le principe de l'utilisateur-payeur semble s'intensifier. Depuisquelquesannées, lesparcsnationaux canadiens imposent un tarif de base à l'entrée des parcs pour tous les visiteurs. Les gestionnaires des parcs provinciaux du Québec s'apprêtent à s'engager dans la même voie. Dep plus, une augmentation de tarification est prévisible pour la pratique d'activités et l'obtention de services. Le désengagement financier de l'Etat aura des effets encore plus dramatiques au moment où les délégataires seront tenus d'investir dans le remplacement des infrastructures vieillissantes des parcs les plus anciens.

En somme, le défi de l'équité sociale pour les générations actuelles et futures demeure un objectif d'actualité et un enjeu majeur pour l'avenir. Les changements de politique risquent de mettre en péril le principe consacré ad'une chance égale pour tous». En plus de sauvegarder l'intégrité écologiquedes ressources, l'enjeuvise également à offrir une wexpérience de pato de qualité aux visiteurs et garantir une accessibilité raisonnable au patrimoine naturel des Québécois.

\section{Interprétation et \\ éthique environnementale}

Tous les rapports internationaux récents, en matière d'environnement et de développement, soulignent l'importance de susciter des changements majeurs dins la façon actuelle de gérer les ressources vivantes en vue d'un développement durable. Pour atteindre cet objectif fondamental, il faut non seulement utiliser un plan d'action détaillé (Action 21, Sommet de la
Terre), mais a ussi le développement d'une éthique environnementale.

La contribution actuelle des pares à cette éthique environnementale se cristallise autour desprogrammesd'interprétation et des événements spéciaux associés. Une étude récente, portant sur l'état de situation de l'interprétation dans le réseau des parcs québécois en 1992-1993 montre que l'on offre des services et des activités d'interprétation dans l'ensemble du réseau (Lebeau, 1993). Douze parcs possèdent uneprogrammation diversifiée, dispensant à la fois des programmes personnalisés et auto-interprétés; trois parcs offrent exclusivement des sentiers d'auto-interprétation. Le degré de développement des infrastructures d'interprétation s'avère toutefois très incigal d'un pare à l'autre. D'autre part, soulignons que la majorité des pares disposent d'un plan d'interprétation. Ce calde de référence est d'autant plus utile que la délégation de gestion en interprétation est de plus en plus fréquente dans les pares quéhécois. En 1992-1993, six pares (Bic, Gispésie, Grands-Jardins, Ile Bonaventure-Rocher-Perçé, JacquesCartier, Saguenay) ont eu recours à ce type de partenariat. Les activités offertes par le biais des contrats d'autorisation sont diversifiées: randonnées d'interprétations et activités d'animation à caractere scientifique, activités d'interprétation reliées à la faune, causerie en soirée, visite de découverte en minibus, croisières et service d'animation.

Pour l'avenir, la contribution des parcs au développement d'une éthique environnementale est appelóe à sintensifier avec l'engagement des gouvernements dans la voie de l'écocivisme. On remarujue également une implication notable des associations professionnelles chargées de la promotion et de la mise en oeuvre de l'écotourisme, dans le développement de codes d'éthique environnementaux (Hawkes et Williams, 1993). Dans cette perspective, lesrésen uxde parcs demeurent des lieux privilégiés pour la dịffusion des messiges de conservation. A partir de l'observation directe d'un phénomène naturel, limité dans l'espace et dans le temps, l'écotourisme suscite une conscientisation des visiteurs à l'environnement global. Cette forme de tourisme amène progressivement les individus à passer de la sensibilisation à l'approfondissement des connaissances, puis de l'appréciation de la nature à un désir de respect et de conserva- tion. A long terme, les effets induits du développement durable et del'écotourisme visent des changements de valeurs, d'attitudes et de comportements.

Dans le futur, les programmes d'interprétation devtont de plus en plus s'ouvrir aux écosystèmes extérieurs des parcs afin de conscientiser et de susciter une responsabilisation des visiteurs face aux enjeux environnementaux régionauxetnationaux. Pour jouer pleinement ce rôle d'éveil à l'environnement global, les gestionnaires de parcs doivent disposer des conditions matérielles adéquates: moyens financiers suffisants, niveaux d'effectifs adéquats, collaboration de partenariat, etc.

\section{Des pares pour le développement}

La stratégie du développement durable propose un autre fil conducteur: la nécessité d'intégrer les considérations environnementales et économiques dans la prise de décisions. On y trouvel'idée que la conservation de l'environnement peut directement contribuer à la croissance économique. Dans le contexte des réseaux de parcs, cette stratégie implique que les territoires protégés ne soient plus considérés comme des sites indépendants des territoiresadjacentset desrégions d'accueil. Au contraire, l'idée edes parcs pour le développements exige la collaboration des gestionnaires au développement local et régional, tout en favorisint l'implication des communautés humaines vivant en périphérie des parcs.

Les parcs, associésà l'industrie touristique, peuvent contribuer à la diversification de ['économie des régions d'accueil. Historiquement, plusieurs régions éloignées se sont appuyées sur une économie rurale et forestière. Le déclin de ces activités traditionnelles présente une menace pour l'avenir. Comme activités complémentaires, les parcs de conservation et l'écotourisme forment un moyen prometteur pour diversifier les sources de revenus des populations locales. De plus, ces nouvelles activités sont susceptibles de contribuer à la réduction du développement économique inégal entre les régions centrales et les régions périphériques. L'implantation de parcs, au sein de régions dévitalisées et éloignées des grands centres, est considéré ì la fois comme un facteur de préservation du patrimoine naturel et culturel des communautés humaines et comme un facteur de rétention des populations locales. A ces premiers effets 
viennent s'ajouter une amélioration de la visibilité régionale, une augmentation des fréquentations touristiques et la stimulation des économies locales.

La complémentarité entre les pares et l'industrie touristique nécessite un arrimage deplusen plus étroit. D'une part, en raison de leur valeur intrinsèque en tant que capital-nature, les parcs jouent un rôle d'attraction. En plus des paysages, on y trouve des infrastructures d'accueil et une offre de services visant à répondre aux besoins immédiats des clientèles. Toutefois, compte tenu de la mission de ces espaces protégés, l'ampleur et l'éventail des installations offertes à l'intérieur des parcs demeurent limités. En conséquence, autour de ces pôles, on assiste à l'émergence d'une demande pour l'implantation d'infrastructures d'hébergement, de restauration et de services associés. Ceteffet d'entraînement peut également avoir pour résultat le développement de produits touristiques complémentaires dans la zone périphérique des parcs et dans les régions d'accueil. La concentration de l'offre touristique, autour de sites à forte valeur attractive, est vivement recherchée par les visiteurs. L'actualisation decette gamme de services, dans le pourtour des parcs, implique l'apport d'investissements privés qui s'additionnent aux premieres immobilisations des parcs.

La situation évolutive, précédemment décrite, illustre une relation de murualisme où deux entités retirent des bénéfices réciproques. La création et la mise en valeur des parcs suscitent, dans bien des cas, l'émergence ou la consolidation del'industrietouristique régionale. En contrepartie, les produits touristiques complémentaires contribuent à former une masse critique suffisante capable de décider les touristes à se déplacer pour visiter les parcs de conservation souvent situés dans des régions éloignées. Cette offredeservices, dela part d'organismes privés, contribue également à réduire les pressions de développement ì l'intérieur même des parcs.

\section{Conclusion}

Depuis les années 1970, le gouvernement du Québec a fait preuve d'efforts considérables. Au plan de l'accessibilité, les parcs de récréation présentent une localisation, une offre de services et une fréquentation remarquables. Les parcs de conservation présentent un fort potentiel de développement pour la création de produits éco- touristiques. Le désengagement de l'État dans le financement et la gestion des parcs laisse entrevoir l'abandon du concept de gérance exclusive des territoires et des services pour tendre vers le nouvenu concept de gestion et de responsibilités partagées. Cette ouverture au partenariat semble s'appuyer sur une combinaison de trois modes de gestion: une gestion publique (préservation et mise en valeur des ressources naturelles des parcs); une gestion mixte(l'Étatoonserveson rôlededéfinisseur d'objectifs et d'évaluateur desactions, alors queles programmes peuvent être gérés par des mandataires); une gestion privée d'activités ou de services à caractère commercial (ententes de délégation de gestion). Une stratégie de promotion globale est en voie d'assurer une meilleure coordination des actions des differents gestionnaires et intervenants touristiques impliqués.

Au plan de la conservation, lesefforts effectués en vue de maintenir la pérennité des ressources naturelles et l'intégrité écologique des territoires méritent une mention honorable(MEF, Stratégiedeconservation et de protection du résenu des parcs québécois, 1994). De façon générale, les menaces internes sont présentement assez bien contrôlées. Par contre, les menaces externes affectant les ressources naturelles des pares ne peuvent faire l'objet de correctifs que dans le cadre d'activités coordonnées en collaboration avecd"autres organismes, tantpublicsqueprivés. D'autre part, il apparaît primordial de compléter le réseau québécois des parcs. La Commission mondiale sur l'environnement et le développement (1987) recommande que les pays réserwont $12 \%$ de leurs terres afin de protéger la diversité des espèces et des écosystèmes de leurs territoires respectifs. Le plan d'action sur les parcs du Québec précise que la création des cinq pares projetés au sud de la prowince et la mise en réserve de 18 territoires au nord du 50 e parallèle pourraient porter de $0,4 \%$ a 3,7 $y_{1}$ la superficie des aires protégées au Québec (MLCP, 1992, p. 21). Les chiffres énoncés illustrent aussi la démesure du défi à affronter pour l'avenir. L'cart demeure considérable entre la superficie proposée par la CMED $(12$ wa) et le pourcentage anticipé de milieux naturels protégés pour le Québec $(3,7 \%)$. L'objectif ré̉liste se situe probablement entre cesdeux chiffres et implique egalement d accroitre le nombre et la superficie des autres types de milieux maturels protégés.
La vision traditionnelle des pares nordaméricains, séparant l"homme et la nature, est en voiedemutation. Les parcs, formant des îlots de conservation isolés, ne répondent plus aux réalités nationales et internationales contemporaines. C'est pourquoi, l'un des défis majeurs des réseaux de parcs, au cours des prochaines années, consisteà passer del'isolation à l'intégration. Cechangement d'orientation implique une approche globale faworisant l'intégration régionale des parcs, autant au plan écologique que socio-économique. Les parcs, fonctionnant comme des entités administratives en autarcie, ont peu d'avenir. Les problématiques et lesenjeux multisectoriels exigent une approche plus conviviale, fondéesur lesprincipes d'interdépendance avec le milieu local et de partenariat à responsabilités partagées. Ce n'est que dans cette perspective que les parcs pourront jouer pleinement leurs rôles et devenir de véritables modèles d'écodéveloppement s'inscrivant dans la Stratégie mondiale de la conservation pour un développement durable.

\section{REFEERENCES}

BARABE, A. (Éd, I, Lojsir et gestion des milieuk naturels profepes. Environnements de loisiv et ditweloponment durabie, Loisir et Societé, vol. 13, no 2 . 1990,568 p

BLANGY, S. (sous la direction). Tounisme et envirome. ment du tourisme de nature a Yecotourisme, Las Cahiers Espaces. Paris, Editions Touristiques Européennes, têrier 1993.

BUSSIERE, G, Le réseau des parcs du Québec: evolution et relation avec le dévelopoement durable. Loisir et Société, vol. 13, no 2 , Université du Loisir et Sociéte, vol. 13, no 2 , Universite du
Québec a Trois-Rivieres, 1990 . pp. 359.377 .

CONSEIL CONSULTATIF CANADIEN DE L'ENVIRONNEMENT, Une vision des zones protégées pour le Canada, Ottawa, Environnement Canada, 1991.

ENVIRONNEMENT CANADA, L'état des parss. Rapport 1990, Le plan vert du Canada. Ottawa, Service des parcs, 1990

FOISY, L Les loisirs at la préservation des parcs nationaux canadiens, Colloque international sur le losir, Université du Quebec a Tross:Fivières, 1994

HAWKES, S. WILLIAMS, P. (EdS)., The Greening of Tourism: From Principles to Practice. A Casebook of Best Envifonmental Practice in Tourism, Simon Fraser University, Centre tor Tourism Policy and Research, 1993.

LALIBERTE ET LANCTOT, Coopers et Lybrand, Le positionnement des pares ot des róserves launiques du Quebec, Québec, MLCP, 1992.

LEBEAU, $L$. Etat do la situation de I'interprétation dans le réseau des parcs québótcois en 1992 1993, Cuébec MLCP, 1993

MINISTERE DE L'ENVIRON NEMENT ETDE LAFAUNE, Stratégie de conservation ot de protection. Reseau des parcs quebecois, Quebec, Direction du plein air et des parcs, $199 \mathrm{~s}$

MINISTERE DU LOISIR, DE LA CHASSE ET DE LA PECHE, Les parcs quóbécois. Plan de promotion 1993-1996, Quebec, Direction du marketing

MINISTERE DU LOISIA, DE LA CHASSE ET DE LA PECHE, La nature en héritage. Plan d'action sur les parcs, Quebec, MLCP. 1992

MINISTERE DU LOISIA, DE LA CHASSE ET DE LA $P E C H E$, Les parcs quebécois. La politique. Quebec, MLCP, 1982.

PARCS CANADA, Principes directeurs et politiques de gestion. Ottawa, Patrimoine canadien, 1994

RICHEZ, G. Parcs nationaux of tourisme en Europe. Pars, Editions l'Harmattan, 1992 ?

SOUACES UNESCO. Tou isme et nature: le face-aface, no 55, Paris, UNESCO, 1994 